\title{
Localization and Test Exponents for Tight Closure
}

\author{
Melvin Hochster \& Craig Huneke
}

Dedicated to William Fulton

\section{Introduction}

We introduce the notion of a test exponent for tight closure and explore its relationship with the problem of showing that tight closure commutes with localization, a long-standing open question. Roughly speaking, test exponents exist if and only if tight closure commutes with localization; mild conditions on the ring are needed to prove this. We give other, independent, conditions that are necessary and sufficient for tight closure to commute with localization in the general case, in terms of behavior of certain associated primes and behavior of exponents needed to annihilate local cohomology. Although certain related conditions (the ones given here are weaker) were previously known to be sufficient, these are the first conditions of this type that are actually equivalent.

The difficult calculation of Section 4 uses associativity of multiplicities and many other tools to show that sufficient conditions for localization to commute with tight closure can be given in which asymptotic statements about lengths of modules defined using the iterates of the Frobenius endomorphism replace the finiteness conditions on sets of primes introduced in Section 3. The result is local and requires special conditions on the rings: one is that countable prime avoidance holds. This is not a very restrictive condition, however; it suffices, for example, for the ring to contain an uncountable field. Countable prime avoidance also holds in any complete local ring. But we also need the existence of a strong test ideal (see the beginning of Section 4). We expect that, in the long run, this condition will also turn out not to be very restrictive: a strong test ideal for a reduced ring is known to exist if every irreducible component of $\operatorname{Spec} R$ has a resolution of singularities obtained by blowing up an ideal that defines the singular locus, and it is expected that this is always true in the excellent case. Moreover, by very recent results, strong test ideals always exist for complete reduced local rings.

We note that the reader may find other results related to localization of tight closure in [AHH; Hu3; K1; K2; LS; V1; V2].

Received March 24, 2000. Revision received April 19, 2000.

The authors were supported in part by grants from the National Science Foundation.

The second author wishes to thank the Max Planck Institute in Bonn and the Fulbright Foundation for partial support of the research for this manuscript. 


\section{Test Exponents and Associated Primes}

Discussion 2.1 (Basic Terminology and Notation). We shall assume throughout that $R$ is a Noetherian ring of positive prime characteristic $p$, although this hypothesis is usually repeated in theorems and definitions. Moreover, because tight closure problems are unaffected by killing nilpotents, we shall assume, unless otherwise specified, that $R$ is reduced. We shall usually assume that the reduced ring $R$ has a test element $c$ (see the discussion in the fourth paragraph of this section). We recall some terminology and notation. We use $R^{\circ}$ to denote the complement of the union of the minimal primes of $R$; hence, if $R$ is reduced then $R^{\circ}$ is simply the multiplicative system of all nonzerodivisors in $R$. We shall write $\boldsymbol{F}^{e}$ (or $\boldsymbol{F}_{R}^{e}$ if we need to specify the base ring) for the Peskine-Szpiro or Frobenius functor from $R$-modules to $R$-modules. This is a special case of the base change functor from $R$-modules to $S$-modules that is simply given by $S \otimes_{R} \_$: in the case of $\boldsymbol{F}^{e}$, the ring $S$ is $R$ but the map $R \rightarrow R$ that is used for the algebra structure is the eth iteration $F^{e}$ of the Frobenius endomorphism, $F^{e}(r)=r^{p^{e}}$. Thus, if $M$ is given as the cokernel of the map represented by a matrix $\left(r_{i j}\right)$, then $\boldsymbol{F}^{e}(M)$ is the cokernel of the map represented by the matrix $\left(r_{i j}^{p^{e}}\right)$. Unless otherwise indicated, $q$ denotes $p^{e}$ where $e \in \mathbb{N}$. For $q=p^{e}$ we have $\boldsymbol{F}^{e}(R / I) \cong R / I^{[q]}$, where $I^{[q]}$ denotes the ideal generated by the $q$ th powers of all elements of $I$ (equivalently, of generators of $I$ ). Note that $\boldsymbol{F}^{e}$ preserves both freeness and finite generation of modules, and it is exact precisely when $R$ is regular (cf. [He; Ku]). If $N \subseteq M$ we write $N^{[q]}$ for the image of $F^{e}(N)$ in $F^{e}(M)$, although it depends on the inclusion $N \rightarrow M$ and not just on $N$. If $u \in M$ then we write $u^{p^{e}}$ for the image $1 \otimes u$ of $u$ in $F^{e}(M)$. With this notation, $(u+v)^{q}=u^{q}+v^{q}$ and $(r u)^{q}=r^{q} u^{q}$ for $u, v \in M$ and $r \in R$.

It is worth noting that, for any multiplicative system $W$ in $R, \boldsymbol{F}_{R_{W}}^{e}\left(M_{W}\right) \cong$ $F_{R}^{e}(M)_{W}$, where ${ }_{W}{ }$ indicates localization with respect to $W$. In fact, for any $R$-algebra $S$, one has that

$$
\boldsymbol{F}_{S}^{e}\left(S \otimes_{R} M\right) \cong S \otimes_{R} \boldsymbol{F}_{R}^{e}(M) .
$$

Furthermore, $N_{W}^{[q]} \subseteq \boldsymbol{F}^{e}(M)_{W}$ may be canonically identified with $\left(N_{W}\right)^{[q]}$.

An element $u \in M$, where $M$ is a finitely generated $R$-module, is in the tight closure $N^{*}$ of $N \subseteq M$ if there exists $c \in R^{\circ}$ such that $c u^{q} \in N^{[q]}$ for all $q=$ $p^{e} \gg 0$. By the right exactness of tensor, $\boldsymbol{F}^{e}(M / N) \cong \boldsymbol{F}^{e}(M) / N^{[q]}$, where $q=$ $p^{e}$. It follows easily that an element of $M$ is in $N^{*}$ if and only if its image in $M / N$ is in $0^{*}$ in $M / N$. Therefore, in considering whether $u \in M$ is in $N^{*}$, we may replace $M$ by a finitely generated free module $G$ mapping onto $M, N$ by its inverse image in $G$, and $u$ by any element of $G$ that maps to $u$.

An element $c \in R^{\circ}$ is called $a$ test element if, whenever $M$ is a finitely generated $R$-module and $N \subseteq M$ is a submodule, $u \in M$ is in the tight closure of $N$ if and only if, for all $q=p^{e}, c u^{q} \in N^{[q]}$. Thus, if the ring has a test element, it "works" in any tight closure test where some choice of $c \in R^{\circ}$ "works". Test elements are also characterized as the elements of $R^{\circ}$ that annihilate $N^{*} / N$ for all submodules $N$ of all finitely generated modules $M$. 
A test element is called locally stable if its image in every local ring of $R$ is a test element (this implies that it is a locally stable test element in every localization of $R$ at any multiplicative system). A test element is called completely stable if its image in the completion of each local ring of $R$ is a test element. A completely stable test element is easily seen to be locally stable. We refer the reader to [AHH, Sec. 2; HH1, Secs. 6, 8; HH2; HH5, Sec. 6] for more information about test elements and to [AHH, Sec. 3] for a discussion of several basic issues related to the localization problem for tight closure.

We note that, by some rather hard theorems, test elements are known to exist. For example, if $R$ is any reduced ring essentially of finite type over an excellent local ring, then $R$ has a test element. In fact, if $c$ is any element of $R^{\circ}$ such that $R_{c}$ is regular (and such elements always exist if $R$ is excellent and reduced), then $c$ has a power that is a completely stable test element. This follows from Theorem 6.1a of [HH5], and we shall make use of this freely throughout.

Another important property of tight closure in characteristic $p$ is that if $R \rightarrow S$ is a ring homomorphism and $u \in N^{*}$ in $M$, then the image $1 \otimes u$ of $u$ in $S \otimes_{R} M$ is in the tight closure, over $S$, of

$$
\operatorname{Im}\left(S \otimes_{R} N \rightarrow S \otimes_{R} M\right)
$$

under very mild assumptions. This phenomenon is referred to as the persistence of tight closure. In particular, by Theorems 6.23 (which, with the same proof, is valid whenever $R$ is essentially of finite type over an excellent local ring-the result is stated only for the case of finite type) and 6.24 of [HH5], if $R$ is essentially of finite type over an excellent local ring, if $S$ has a completely stable test element, or if $R^{\circ}$ maps into $S^{\circ}$ (e.g., if $R \rightarrow S$ is an inclusion of domains or is flat), then one has persistence of tight closure for the ring homomorphism $R \rightarrow S$.

Definition 2.2. Let $R$ be a reduced Noetherian ring of positive prime characteristic $p$. Let $c$ be a fixed test element for $R$, and let $N \subseteq M$ be a pair of finitely generated $R$-modules. We shall say that $q=p^{e}$ is a test exponent for $c, N, M$ if $u \in N^{*}$ whenever $c u^{Q} \in N^{[Q]}$ and $Q \geq q$. In case $N$ is an ideal it is usually assumed that $M=R$, and in that case we speak of a test exponent for $c, I$, with $R$ understood to be the ambient module for $I$.

It is not at all clear whether to expect test exponents to exist. In this paper we shall prove (roughly speaking) that test exponents exist, in general, if and only if tight closure commutes with localization. The question of whether tight closure commutes with localization is open in general, but it is known in many important special cases, and thus our results imply that test exponents do exist rather often. We expect this notion to be of great importance because, whenever one can compute what the test exponent is, one obtains an effective test for tight closure.

We are also hopeful that focusing attention on the problem of the existence of test exponents may lead to a solution of the localization problem for tight closure. We want to point out that, if tight closure commutes with localization, then it commutes with arbitrary smooth base change; for a precise statement, see [HH5, Thm. 7.18a]. 
To demonstrate one connection, we prove the easier half of the result at once (this half is implicit in [McD]).

Proposition 2.3. Let $R$ be a reduced Noetherian ring of positive prime characteristic $p$ and let $N \subseteq M$ be finitely generated $R$-modules. Let $c$ be a locally stable test element for $R$. If there is a test exponent for $c, N, M$ then, for every multiplicative system $W$ of $R,\left(N_{W}\right)^{*}$ in $M_{W}$ over $R_{W}$ is $\left(N^{*}\right)_{W}$ (i.e., tight closure for the pair $N \subseteq M$ commutes with localization).

Proof. The only problem is to show that if $u \in M$ and $u / 1 \in\left(N_{W}\right)^{*}$ then $u \in$ $\left(N^{*}\right)_{W}$ (any element of $M_{W}$, after multiplication by a suitable unit, is in the image of $M$ ). Let $q$ be a test exponent for $c, N, M$. Then we can choose $c u^{q} / 1 \in N_{W}^{[q]}$ and we can choose $f \in W$ such that $f c u^{q} \in N^{q}$, so $c(f u)^{q} \in N^{[q]}$. But then $f u \in$ $N^{*}$ and so $u \in\left(N^{*}\right)_{W}$.

What is much less obvious is that a converse holds.

THEOREM 2.4. Let $R$ be a reduced Noetherian ring of positive prime characteristic $p$, and let $N \subseteq M$ be a pair of finitely generated $R$-modules. Suppose that, for every prime $Q \in \operatorname{Ass}\left(M / N^{*}\right),\left(N_{Q}\right)^{*}=\left(N^{*}\right)_{Q}$. Then, for every test element $c \in R^{\circ}$ such that $c$ is a test element in each of the rings $R_{Q}$ for every associated prime $Q$ of $M / N$, there is a test exponent for $c, M, N$. In particular, if $\left(N_{Q}\right)^{*}=$ $\left(N^{*}\right)_{Q}$ for all associated primes $Q$ of $M / N^{*}$ and if $c$ is a locally stable test element for $R$, then $c, N, M$ has a test exponent.

Thus, if tight closure commutes with localization for the pair $N \subseteq M$ at associated primes of $M / N^{*}$ and if $R$ has a locally stable test element, then tight closure commutes with localization in general for the pair $N \subseteq M$.

We postpone the proof until we have established some preliminary results that make the argument transparent (it is given immediately following the proof of Theorem 2.7).

Proposition 2.5. Let $R$ be a reduced Noetherian ring of positive prime characteristic $p$, and let $M$ be a finitely generated $R$-module. Let $N, N^{\prime}$, and $N_{i}$ (where $i$ varies in an index set) be submodules of $M$. Let $c \in R^{\circ}$ be a test element.

(a) $q=p^{e}$ is a test exponent for $c, N, M$ if and only if it is a test exponent for $c, 0, M / N$.

(b) If $q=p^{e}$ is a test exponent for $c, N, M$ then so is every larger power of $p$.

(c) If $N \subseteq N^{\prime} \subseteq N^{*}$ and $q$ is a test exponent for $c, N^{\prime}, M$, then $q$ is a test exponent for $c, N, M$. In particular, this holds when $N^{\prime}=N^{*}$.

(d) If $N \subseteq N^{\prime} \subseteq N^{*}$, $d$ is a test element, and $q$ is a test exponent for $c d, N, M$, then $q$ is a test exponent for $c, N^{\prime}, M$.

(e) If $N_{1}$ and $N_{2}$ have the same tight closure in $M$ and if $W$ is a multiplicative system consisting of test elements, then $c_{1}, N_{1}, M$ has a test exponent for every choice of test element $c_{1} \in W$ if and only if $c_{2}, N_{2}, M$ has a test exponent for every choice of test element $c_{2} \in W$. 
(f) If $q$ is a test exponent for $c, N_{i}, M$ for every index $i$ (the index set may be infinite), if every $N_{i}$ is tightly closed, and if $N=\bigcap_{i} N_{i}$, then $q$ is a test exponent for $c, N, M$.

(g) If $N_{1}, \ldots, N_{h}$ are submodules of $N$ such that there is a test exponent for each of $c, N_{i}, M$ for $1 \leq i \leq h$, then there is a test exponent for $c, \bigcap_{i} N_{i}, M$.

Proof. Part (a) is immediate from the definitions of test exponent and tight closure, while (b) is immediate from the definition of test exponent. In part (c), since $N^{\prime}$ and $N$ have the same tight closure, it follows that if $q$ is a test exponent for $c, N^{\prime}, M$ then $c u^{Q} \in N^{[Q]}$ for $Q \geq q$ implies that $c u^{Q} \in N^{\prime[Q]}$, and so $u \in N^{\prime *}=$ $N^{*}$. For part (d): if $q$ is a test exponent for $c d, N, M$, if $Q \geq q$, and if $c u^{Q} \in$ $N^{\prime[Q]}$, then (since $N^{\prime}$ is in the tight closure of $N$ ) we have that $N^{\prime[Q]}$ is in the tight closure of $N^{[Q]}$ and so $c d u^{Q} \in N^{[Q]}$; hence we can conclude that $u \in N^{*}=N^{\prime *}$. For part (e), we might as well assume that $N_{2}$ is the tight closure of $N_{1}$; it suffices to compare each with its tight closure. The result is immediate from the combination of parts (c) and (d). Part (f) is immediate from the definition, for if $c u^{Q} \in$ $N^{[Q]}$ then $c u^{Q} \in N_{i}^{[Q]}$ for all $i$, and so $u \in N_{i}$ for all $i$, which shows that $u \in N$. Part (g) follows from (b) and (f): we may use the supremum of the finitely many test exponents for the various $N_{i}$.

Proposition 2.6. Let $R$ be a reduced Noetherian ring of positive prime characteristic $p$. Let $N \subseteq M$ be finitely generated modules such that $M / N$ has a unique associated prime $P, N$ is tightly closed in $M$, and $N_{P}$ is tightly closed in $M_{P}$ over $R_{P}$. Let $c \in R^{\circ}$ be any test element for $R$ that is also a test element in $R_{P}$. Then there is a test exponent for $c, N, M$.

Proof. We first consider the case where $P$ is maximal and $R=R_{P}$. Let $N_{e}$ denote the set of elements $u \in M$ such that $c u^{q} \in\left(N^{[q]}\right)^{\boldsymbol{F}}$, where $q=p^{e}$ and $\boldsymbol{F}$ indicates Frobenius closure in $M^{[q]}=\boldsymbol{F}^{e}(M)$. Clearly, $N^{*} \subseteq N_{e}$ for every $e$. We claim that $N_{e+1} \subseteq N_{e}$, for if $c u^{p q} \subseteq N^{[p q]^{F}}$ then $c^{q^{\prime}} u^{p q q^{\prime}} \in N^{\left[p q q^{\prime}\right]}$, which certainly implies that $c^{p q^{\prime}} u^{p q q^{\prime}} \in N^{\left[p q q^{\prime}\right]}$, and this shows that $c u^{q} \in N^{[q]}{ }^{\boldsymbol{F}}$, as required. Thus, this sequence of modules is eventually constant, since $M / N$ has finite length in this case. But once $N_{e}=N_{e+1}=\cdots$, the common value must be $N^{*}$, for if $u$ is in all of these then $c u^{Q} \in N^{[Q]}{ }^{F} \subseteq N^{[Q]^{*}}$ for all $Q \geq p^{e}$ and so $c^{2} u^{Q} \in N^{[Q]}$ for all $Q \geq p^{e}$, as required. Then $c u^{\bar{q}^{\prime}} \in N^{\left[q^{\prime}\right]}$ for $q^{\prime} \geq p^{e}$ implies that $u \in N_{e}=N^{*}$, as required.

In the general case, note that the hypothesis is stable when we localize at $P$ and so, by the case already proved, there exists $q^{\prime}$ such that $c u^{q^{\prime}} / 1 \in N_{P}{ }^{\left[q^{\prime}\right]}$ implies that $u / 1 \in N_{P}$. But then, since $c u^{q^{\prime}} \in N^{\left[q^{\prime}\right]}$ is preserved when we localize at $P$, we have that this implies that $u$ is in the contraction of $N_{P}$ to $M$, and since $M / N$ is $P$-coprimary, this implies that $u \in N$.

TheOREM 2.7 (Existence of Primary Decompositions That Respect Tight Closure). Let $R$ be a reduced Noetherian ring of positive prime characteristic $p$, and suppose that $R$ has a test element. Let $N \subseteq M$ be finitely generated modules and 
suppose that $R_{P}$ has a test element (e.g., is excellent) for every associated prime of $M / N$. Suppose that $N$ is tightly closed in $M$ and that $N_{P}$ is tightly closed in $M_{P}$ for every associated prime $P$ of $M / N$.

Then $N$ has a primary decomposition in $M$ in which, for every associated prime $P$ of $M / N$, the P-primary component is tightly closed and remains tightly closed (in $M_{P}$, over $R_{P}$ ) after localization at $P$.

Proof. It suffices to construct such a primary decomposition for $N_{P} \subseteq M_{P}$ over $R_{P}$ for each associated prime $P$ of $M / N$. Take all these submodules of $M_{P}$ and contract them to $M$, with $P$ varying among the associated primes of $M / N$. (Note that the contraction of a tightly closed submodule of $M_{P}$ to $M$ is tightly closed in $M$ : this is a consequence of the persistence of tight closure, which is automatic for the flat homomorphism $R \rightarrow R_{P}$.) When there are several primary components for the same prime $Q$ (each tightly closed, each remaining tightly closed over $R_{Q}$ ), intersect them all.

This gives a primary decomposition of $N$ with the required properties. To see that it is, in fact, a primary decomposition, call the intersection $N^{\prime}$. If $N^{\prime} / N \neq$ 0 then it contains a nonzero element whose annihilator is an associated prime of $M / N$, and this remains true after localizing at that associated prime, which gives a contradiction.

Thus, there is no loss of generality in assuming that $(R, P)$ is local, that $P$ is an associated prime of $M / N$, and that we have solved the problem of constructing suitable primary components after localizing at any of the other associated primes (by induction on the dimension of $R_{P}$ ). Thus, we may give a primary decomposition over $R_{Q}$ for every associated prime $Q$ strictly contained in $P$ and then intersect the contractions of all the modules occurring as before. Call the intersection of these other primary components $H$. Thus, $H$ has a primary decomposition using modules that are (i) primary for the other associated primes of $M / N$ and (ii) tightly closed and remain so upon localization at the respective associated primes.

If we localize at any element of $P$ then only the other associated primes remain. Hence $H / N$ is killed by a power of $P$ and is a module of finite length.

Now consider the descending chain of submodules $\left(N+P^{n} M\right)^{*}=N_{n}$, which are tightly closed submodules of $M$ containing $N$. Then $\left(N_{n} \cap H\right) / N$ is contained in $H / N$ and so the chain $\left(N_{n} \cap H\right) / N$ is eventually stable, which means that $N_{n} \cap H$ is eventually stable. But the intersection of the $N_{n}$ is $N$, because $N$ is tightly closed and $R$ has a test element. (If $u \in N_{n}$ for all $n$ and if $c$ is the test element, then for all $q, c u^{q} \in\left(N+P^{n} M\right)^{[q]} \subseteq N^{[q]}+P^{n} \boldsymbol{F}^{e}(M)$, and since $N^{[q]}$ is $P$-adically closed in $\boldsymbol{F}^{e}(M)$, we have that $c u^{q} \in N^{[q]}$ for all $q$ and so $u \in N^{*}=N$, as required.) The stable value of $N_{n} \cap H$ must be the same as $\bigcap_{n}\left(N_{n} \cap H\right)=\left(\bigcap_{n} N_{n}\right) \cap H=$ $N \cap H=N$. Thus $N_{n} \cap H=N$ for all $n \gg 0$, and we may use $N_{n}$ for any sufficiently large $n$ as the required tightly closed $P$-primary component.

Proof of Theorem 2.4. For the first statement: by Theorem 2.7 coupled with Proposition 2.6, $N$ is a finite intersection of submodules $N_{i}$ of $M$ such that there is a 
test exponent for $c, N_{i}, M$; the result is then immediate from parts (c) and ( $\mathrm{g}$ ) of Proposition 2.5.

For the second statement: choose a test element of $R$ and, for each associated prime $Q$ of $M / N^{*}$, an element of $R^{\circ}$ that maps to a test element for $R_{Q}$. The product will be a test element $c$ of $R$ that is also a test element for every $R_{Q}$, so $c, N, M$ must have a test exponent.

COROLlaRY 2.8. Let $R$ be a reduced Noetherian ring of positive prime characteristic $p$, and suppose that $R$ has a locally stable test element. Let $N \subseteq M$ be finitely generated $R$-modules and assume in addition that there is a submodule $N^{\prime} \subseteq N^{*}$ such that $N_{Q}^{\prime}=N_{Q}$ at all associated primes $Q$ of $M / N^{*}$ and the tight closure of $N^{\prime}$ does commute with localization. Then the tight closure of $N$ commutes with localization.

Proof. By Theorem 2.4, it suffices to prove that $\left(N^{*}\right)_{Q}=\left(N_{Q}\right)^{*}$ for all primes $Q$ that are associated to $N^{*}$. Fix such a $Q$. Then $\left(N_{Q}\right)^{*}=\left(N_{Q}^{\prime}\right)^{*}=\left(N^{\prime *}\right)_{Q} \subseteq$ $\left(N^{*}\right)_{Q}$.

COROLlary 2.9. Let $R$ be a reduced Noetherian ring of positive prime characteristic $p$ and suppose that $R$ has a locally stable test element. Suppose that $I \subseteq$ $R$ is generically, a complete intersection (i.e., there is an ideal $I^{\prime}$ generated by a regular sequence such that $I_{P}=I_{P}^{\prime}$ for every minimal prime $P$ of $\left.I\right)$ and that $I^{*}$ has no embedded primes. Then $\left(I_{W}\right)^{*}=\left(I^{*}\right)_{W}$ for all multiplicatively closed sets $W$.

Proof. This follows immediately from Corollary 2.8 and the fact that localization commutes with tight closure for ideals generated by regular sequences (see [HH5, Thm. 4.5]).

REMARK. Corollary 2.8 can be combined with numerous other theorems on when tight closure commutes with localization to give other results similar to Corollary 2.9. For example, if $R$ is a domain of acceptable type in the sense of [AHH, p. 87] (a mild condition satisfied by homomorphic images of Cohen-Macaulay rings and by algebras essentially of finite type over an excellent local ring), then in Corollary 2.9 we need only the ideal $I^{\prime}$ to be of height $d$ and generated by $d$ elements (cf. [AHH, Thm. 8.3b, p. 112]). Note that if $R$ is not a domain then the result is valid if one has the required hypotheses modulo every minimal prime.

COROLlaRY 2.10. Let $R$ be a reduced Noetherian ring of positive prime characteristic $p$ with a locally stable test element. If $\left(J_{Q}\right)^{*}=\left(J^{*}\right)_{Q}$ for every primary ideal $J$ with radical $Q$, then $\left(I_{W}\right)^{*}=\left(I^{*}\right)_{W}$ for every ideal I such that $I^{*}$ has no embedded primes and for every multiplicatively closed set $W$.

Proof. First note that the hypothesis implies that $\left(J_{W}\right)^{*}=\left(J^{*}\right)_{W}$ for every primary ideal $J$ and for every multiplicative set $W$.

Fix an arbitrary ideal $I$ with $I^{*}$ unmixed. Let $Q_{1}, \ldots, Q_{n}$ be the associated primes of $I^{*}$. These are the same as the minimal primes of $I^{*}$, and since $I$ and $I^{*}$ 
have the same radical, they are precisely the minimal primes of $I$. Let $J_{i}$ be the primary component of $I$ corresponding to $Q_{i}$. Since the $Q_{i}$ are minimal over $I$, this component is uniquely determined. By Theorem 2.4, it is enough to prove that $\left(I^{*}\right)_{Q_{i}}=\left(I_{Q_{i}}\right)^{*}$ for each $Q_{i}$. We have that $\left(I_{Q_{i}}\right)^{*}=\left(J_{i}^{*}\right)_{Q_{i}}$. It suffices to prove that $J_{i}^{*} \subseteq\left(I^{*}\right)_{Q_{i}}$, for then $\left(I_{Q_{i}}\right)^{*}=\left(J_{i}^{*}\right)_{Q_{i}} \subseteq\left(I^{*}\right)_{Q_{i}}$. Choose $u \in I: J_{i}$ such that $u \notin Q_{i}$, and let $x \in J_{i}^{*}$. For some $d \in R^{\circ}$ and for all large $q$, we have $d x^{q} \in$ $\left(J_{i}\right)^{[q]}$. Multiplying by $u^{q}$ gives that $d(u x)^{q} \in I^{[q]}$, proving that $u x \in I^{*}$ and $x \in$ $\left(I^{*}\right)_{Q_{i}}$, as required.

Discussion 2.11. Let $R$ be a reduced Noetherian ring of positive prime characteristic $p$. Given the results of this section, it is natural to ask how the associated primes of $I \subseteq R$ and of $I^{*}$ are related as well as how, more generally, the associated primes of $M / N$ and $M / N^{*}$ are related when $N \subseteq M$, a finitely generated $R$-module. Note that although the minimal primes are the same, there is, in general, no comparison, in either direction, for the other associated primes. The examples that follow will show this even in the case of ideals. (Note: To see that a minimal prime $Q$ of $M / N$ is still in the support of $M / N^{*}$ we may localize at that prime, since $\left(N_{Q}\right)^{*} \supseteq\left(N^{*}\right)_{Q}$, and then use the fact that $(M / N)_{Q}$, if nonzero, maps on $R_{Q} / Q R_{Q}$.) Embedded primes of $I$ may fail to be associated primes of $I^{*}$, and embedded primes of $I^{*}$ may fail to be associated primes of $I$. In Example 2.12, $I$ has the maximal ideal as an embedded prime but $I^{*}$ does not; in Example 2.13, $I$ is unmixed but $I^{*}$ has the maximal ideal as an embedded prime.

EXAMPLE 2.12. Let $R$ be a normal, 3-dimensional local ring of positive prime characteristic that is not Cohen-Macaulay. (For definiteness, one may take the Segre product of a homogeneous coordinate ring of an elliptic curve, e.g., $K[x, y, z] /\left(x^{3}+y^{3}+z^{3}\right)$, with $K$ a field of positive characteristic different from 3 , with $K[u, v]$, a homogeneous coordinate ring for $\mathbb{P}_{K}^{1}$, and localize at the irrelevant ideal, i.e., at the unique maximal homogeneous ideal.) Let $f, g$ be part of a system of parameters. Since $R$ is not Cohen-Macaulay, the maximal ideal is an embedded prime of $I=(f, g) R$. By the colon-capturing property of tight closure (see e.g. [HH6, Thm. 1.7.4]), a third parameter for $R$ is not a zerodivisor on $I^{*}$ and so the maximal ideal of $R$ is not an associated prime of $I^{*}$. There are similar examples in all dimensions.

ExAmPLe 2.13. Let $K$ be a field of positive prime characteristic $p \neq 3$. Take

$$
R=K[X, Y, U, V] /\left(X^{3} Y^{3}+U^{3}+V^{3}\right)=K[x, y, u, v] .
$$

Then $R$ is geometrically normal ( $p \neq 3$ ) since the partial derivatives of the defining polynomial include $3 U^{2}$ and $3 V^{2}$, which form a regular sequence in the ring. Let $I=\left(u, v, x^{3}\right) R$. By the persistence of tight closure (cf. the last paragraph of Section 2.1) and the fact that $z^{2}$ is in $(u, v)^{*}$ in $K[z, u, v] /\left(z^{3}+u^{3}+v^{3}\right)$ (see e.g. the beginning of Sec. 4 in [H1]), we have that $x^{2} y^{2}$ is in the tight closure of $(u, v)$ and hence in $I^{*}$. Now $R / I$ is isomorphic with $K[x, y] /\left(x^{3}\right)$ and so $I$ is unmixed; $I^{*}$ contains $x^{2} y^{2}$, so that both $y^{2}$ and $x$ multiply $x^{2}$ into $I^{*}$ (as well as $u$ and $v$, of course). This will show that $R / I^{*}$ has $m=(x, y, u, v)$ as an embedded prime, 
provided that $x^{2}$ is not in $I^{*}$. But this is true even if we kill $u, v$, and $y$, and tight closure persists under homomorphisms for affine algebras (cf. once again the last paragraph of Discussion 2.1).

We next observe that if tight closure commutes with localization after a faithfully flat extension then it commutes with localization.

Proposition 2.14. Let $R$ be a reduced Noetherian ring of positive prime characteristic $p$ such that $R$ has a completely stable test element. Let $R \rightarrow S$ be faithfully flat, and let $N \subseteq M$ be finitely generated $R$-modules. Let $W$ be a multiplicative system in $R$. If tight closure commutes with localization for the pair $S \otimes_{R} N \subseteq$ $S \otimes_{R} M$ and the multiplicative system that is the image of $W$ in $S$, then it commutes with localization for the pair $N \subseteq M$ and the multiplicative system $W$.

Proof. It suffices to show that, if $u \in M$ is such that $u / 1$ is in the tight closure of $N_{W}$ in $M_{W}$, then $u$ is in $\left(N^{*}\right)_{W}$. But then $1 \otimes u$ is in the tight closure of $S_{W} \otimes_{R} N$ in $S_{W} \otimes_{R} M$ over $S_{W}$, by the persistence of tight closure (this is trivial in the flat case), and by our hypothesis this implies that $1 \otimes u$ is in $\left(S \otimes_{R} N\right)^{*}$ in $\left(S \otimes_{R} M\right)^{*}$ over $S$. This implies that $u$ is in $N^{*}$, by [H2, Cor. 8.8, p. 143].

REMARK 2.15. The conclusion of Proposition 2.14 is valid under a substantial weakening of the hypothesis on the homomorphism $R \rightarrow S$. Instead of being faithfully flat, it suffices if it preserves height (in the sense of condition $(*)$ of [H2, Cor. 8.8]) and persistence of tight closure holds. The proof is the same.

COROLlaRY 2.16. Let $R$ be a reduced ring and $K$ a field such that $R$ satisfies at least one of the following conditions:

(1) $R$ is finite type over $K$;

(2) $R$ is essentially of finite type over $K$;

(3) $R$ is an excellent local ring with residue field $K$;

(4) $R$ is of finite type over an excellent local ring with residue field $K$;

(5) $R$ is essentially of finite type over an excellent local ring with residue field $K$.

Then $R$ has a faithfully flat extension $S$ that satisfies the same condition but such that $K$ is uncountable and $F$-finite and, in cases (3)-(5), such that the local ring is complete.

Hence, the question of whether tight closure commutes with localization for any of the rings in the five classes just described can be reduced to a corresponding case where the ring contains an uncountable field and is F-finite and, in cases (3)-(5), where the local ring is complete as well.

Proof. The last statement follows from the next-to-last statement by Proposition 2.14. We may trivially replace the local ring by its completion in (3)-(5). The statements about rings essentially of finite type follow from the corresponding statements for rings of finite type. In cases (3)-(5) choose a coefficient field, and let $L$ be a field obtained by adjoining uncountably many indeterminates to it (or simply to $K$ in case (1)). Replace the local ring by its complete tensor product 
with $L$ in cases (3) and (4). One may then use the $\Gamma$ construction of [HH5, Sec. 6] to make an excellent, faithfully flat extension of the local ring such that the residue field is $F$-finite, without losing the property that its tensor product with $R$ over the original local ring is reduced. Finally, one may replace this local ring by its completion. Case (1) is simply the special case where the local ring has dimension 0 .

Note that there are several implications among the conditions listed in Corollary 2.16 (e.g., (5) $\Rightarrow(4) \Rightarrow$ (3) and (4) $\Rightarrow(2) \Rightarrow(1)$ ). We have stated the result as we did because it contains the information that, whichever one of these conditions holds, that particular condition can be preserved as one modifies $R$ to contain an uncountable field.

REMARK 2.17. Let $R$ be any ring containing an uncountable field $K$, and let $I$ be a finitely generated ideal of $R$. Let $\left\{I_{n}: n \in \mathbb{N}\right\}$ be a countable family of ideals of $R$ whose union contains $I$. Then $I$ is contained in one of $I_{n}$. To see this, let $V$ be the finite-dimensional $K$-vector space spanned over $K$ by a finite set of generators for $I$. Then $V$ is covered by the vector spaces $I_{n}$ as $n$ varies, and it suffices to show that one of these is $V$, for if $V \subseteq I_{n}$ then $I \subseteq I_{n}$. Thus, we have reduced to a question purely about vector spaces. But the result for vector spaces is clear if the dimension of $V$ is at most 1 , and it follows at once by induction from the fact that $V$ has uncountably many mutually distinct subspaces of codimension 1 , each of which (by the induction hypothesis) will be contained in at least one of the $I_{n}$. It follows that at least two of these are contained in the same $I_{n}$, and this forces $V \subseteq I_{n}$.

Thus, if a Noetherian ring $R$ contains an uncountable field and if the ideal $I$ is not contained in any of the countably many ideals $I_{n}$, then $I$ has an element that is not in any of the $I_{n}$. If this property holds when the $I_{n}$ are prime, we say that $R$ has countable prime avoidance.

When coupled with Corollary 2.16, this discussion shows that, in the main cases, the question of whether tight closure commutes with localization reduces to the case where the ring has countable prime avoidance. We assume countable prime avoidance in the main result of Section 4.

Remark 2.18. We also want to remark that every Noetherian ring $R$ of positive prime characteristic $p$ has a faithfully flat Noetherian extension containing an uncountable field $K$. For any ring $R$, if $T$ is a set of indeterminates then let $R(T)$ denote the localization of the polynomial ring $R[T]$ in these indeterminates at the multiplicative system of all polynomials whose coefficients generate the unit ideal; $R(T)$ is easily seen to be faithfully flat over $R$. Evidently, if $R$ contains any field $K$ (e.g. $\mathbb{Z} / p Z$ ) then $R(T)$ contains $K(T)$, and so if $R$ has characteristic $p$ and $T$ is uncountable then $R(T)$ contains the uncountable field $K(T)$. But, for any Noetherian ring $R, S=R(T)$ is Noetherian.

(It is easy to see that expansion and contraction gives a bijection between maximal ideals of $R$ and those of $S$. It suffices if every prime $P$ of $S$ is finitely generated. 
Let $W$ run through the finite subsets of $T$ and let $P_{W}$ denote the contraction of $P$ to $R(W) \subseteq R(T)$. It is clear that $R(W)$ is Noetherian and so each $P_{W}$ is a finitely generated prime ideal of $R(W)$. Now $P_{W} S$ is prime in $S$, since $S$ is obtained from $R(W)$ by adjoining indeterminates and localizing, and $P_{W} S \subseteq P_{W^{\prime}} S$ if $W \subseteq W^{\prime}$. The prime $P$ is the union of the $P_{W} S$ as $W$ varies. We claim that $P_{W} S=P$ for any sufficiently large choice of $W$. The point is that $P$ is contained in a maximal ideal of $S$, say $m S$, where $m$ is a maximal ideal of $R$ with, say, $n$ generators. Then, for all $W, m S$ lies over $m R(W)$, which has height at most $n$. From this it follows that if one has a chain of the form $P_{W_{0}} S \subset \cdots \subset P_{W_{h}} S$ in which the inclusions are strict, then $h \leq n$, since the inclusions will also be strict in $P_{W_{0}} R(W) \subset \cdots \subset$ $P_{W_{h}} R(W)$ for some sufficiently large $W \supseteq W_{h}$, and this contradicts the fact that $m R(W)$ has height at most $n$.)

REMARK 2.19. We note that, by [B, Lemma 3], countable prime avoidance holds in any complete local ring.

\section{Necessary and Sufficient Conditions for Localization}

In this section we give another necessary and sufficient condition for the tight closure of an ideal to commute with localization. Several sufficient conditions have been given in previous papers. Most notable among these were that tight closure commutes with localization provided that the following two conditions hold (cf. [K1; K2]):

(1) for every ideal $I$, the union, over $q$, of the sets of maximal associated primes of $\left(I^{[q]}\right)^{*}$ is a finite set;

(2) for every prime ideal $P$, there exists a positive integer $k$ such that, for all $q=p^{e}$

$$
P^{k q} H_{P}^{0}\left(R /\left(\left(I^{[q]}\right)^{*}\right)_{P}\right)=0 .
$$

However, these conditions are not known to be necessary. In Theorem 3.5 we give a pair of conditions that characterize precisely when tight closure commutes with localization for an ideal $I$. Conditions like our $\left(\mathrm{C} 2^{*}\right)$ discussed in the sequel are clearly weaker than condition (2) above. Our condition (C1) is reminiscent of condition (1) above in that it asserts the finiteness of certain sets of primes, but it is not immediately apparent how to compare it to (1) directly.

Several preliminaries are needed before stating our main result, Theorem 3.5. In particular, we need to identify certain sets of primes in $R$; these are characterized by several equivalent conditions in the next result.

Proposition 3.1. Let $R$ be a reduced Noetherian ring of positive prime characteristic $p$ with at least one locally stable test element. Let $I \subseteq R$ and $x \in R$ be fixed. Then the following conditions are equivalent for a prime ideal $Q$.

(1) For some (equivalently, every) locally stable test element $c \in R^{\circ}, Q$ is minimal over infinitely many of the ideals $I^{[q]}: c x^{q}$.

(2) For some (equivalently, every) locally stable test element $c \in R^{\circ}, Q$ is minimal over $I^{[q]}: c x^{q}$ for all $q \gg 0$. 
(3) For some (equivalently, every) locally stable test element $c \in R^{\circ}, Q$ is minimal over infinitely many ideals $\left(I^{[q]}\right)^{*}: c x^{q}$.

(4) For some (equivalently, every) locally stable test element $c \in R^{\circ}, Q$ is minimal over $\left(I^{[q]}\right)^{*}: c x^{q}$ for all $q \gg 0$.

(5) $x \notin\left(I_{Q}\right)^{*}$ and $x \in\left(I_{P}\right)^{*}$ for all $P \subsetneq Q$.

Proof. Fix a single locally stable test element $c$. It will suffice to show that the conditions in (1)-(4) are equivalent to the condition in (5) for that one choice of $c$, since (5) does not refer to $c$. Thus, throughout the rest of the proof, $c$ is a fixed locally stable test element and, when we refer to conditions (1)-(4), we are referring to them for that single fixed test element $c$.

Clearly (2) $\Rightarrow$ (1) and (4) $\Rightarrow(3)$.

We next prove that:

(*) If $Q$ is minimal over $I^{\left[q_{i}\right]}: c x^{q_{i}}$ for all $i \geq 1$ then it is minimal over $\left(I^{\left[q_{i}\right]}\right)^{*}$ : $c x^{q_{i}}$ for all $i \gg 0$.

Once we have shown this, it follows at once that (2) $\Rightarrow(4)$ and (1) $\Rightarrow(3)$. Hence, once we establish $(*)$, we need only show that $(5) \Rightarrow(2)$ and $(3) \Rightarrow(5)$ to complete the argument.

Suppose that $(*)$ is false. Since $I^{\left[q_{i}\right]}: c x^{q_{i}} \subseteq\left(I^{\left[q_{i}\right]}\right)^{*}: c x^{q_{i}}, Q$ will be minimal over every $\left(I^{\left[q_{i}\right]}\right)^{*}: c x^{q_{i}}$ that it contains. Suppose that $Q$ does not contain $\left(I^{\left[q_{i}\right]}\right)^{*}: c x^{q_{i}}$ for infinitely many $i$. After localizing at $Q$, we have that $c x^{q_{i}} \in$ $\left(\left(I^{\left[q_{i}\right]}\right)^{*}\right)_{Q}$, which implies that $c^{2} x^{q_{i}} \in\left(I^{\left[q_{i}\right]}\right)_{Q}$ for infinitely many $i$. This shows that $x \in\left(I_{Q}\right)^{*}$ (cf. [HH2, Lemma 8.16, p. 79]). But then $c x^{q} \in I_{Q}^{[q]}$ for all large $q$, proving $I^{[q]}: c x^{q} \nsubseteq Q$ for all large $q$. This contradiction finishes the proof of $(*)$.

Next we prove that $(3) \Rightarrow(5)$. Since $Q$ is minimal over infinitely many of the ideals $\left(I^{[q]}\right)^{*}: c x^{q}$, it follows that $Q$ contains infinitely many of the ideals $I^{[q]}$ : $c x^{q}$ and hence $x \notin\left(I_{Q}\right)^{*}$. Let $P \subsetneq Q$. After localizing at $P$, the assumption guarantees that $c x^{q} \in\left(\left(I^{[q]}\right)^{*}\right)_{P}$ for infinitely many values of $q$, forcing $c^{2} x^{q} \in\left(I^{[q]}\right)_{P}$ for the same values of $q$. As before (cf. [HH2, Lemma 8.16, p. 79]), this implies that $x \in\left(I_{P}\right)^{*}$.

Finally, assume (5). First observe that $Q$ must contain $I^{[q]}: c x^{q}$ for all $q \gg 0$. If not, then (after localizing at $Q$ ) we obtain that $x \in\left(I_{Q}\right)^{*}$, a contradiction. Next, suppose that $P \subsetneq Q$. By assumption, $x \in\left(I_{P}\right)^{*}$; this means that, for all $q$, there exist elements $w_{q} \notin P$ such that $w_{q} c x^{q} \in I^{[q]}$. Then $I^{[q]}: c x^{q} \nsubseteq P$ for all $q$. It follows that $Q$ is minimal over $I^{[q]}: c x^{q}$ whenever it contains $I^{[q]}: c x^{q}$.

DEFINITION 3.2. Let $R$ be a reduced Noetherian ring of positive prime characteristic $p$ having at least one locally stable test element. Let $I \subseteq R$ be an ideal, and let $x \in R$. The primes satisfying the equivalent conditions of (3.1) we call the stable primes associated to $I$ and $x$, and we denote by $T_{I}(x)$ the set of stable primes associated to $I$ and $x$. Let $T_{I}=\bigcup_{x \in R} T_{I}(x)$.

For a fixed ideal $I$ we consider the following two conditions:

(C1) for every $x \in R$, the set $T_{I}(x)$ is finite;

$\left(\mathrm{Cl}^{*}\right)$ the set $T_{I}$ is finite. 
For a fixed ideal $I$ and locally stable test element $c$, we also consider the following two conditions:

(C2) for every $x \in R$, if $Q \in T_{I}(x)$ then there exists an integer $N$, possibly depending on $Q$, such that $Q^{N q} \subseteq\left(I^{[q]}: c x^{q}\right)_{Q}$ for all $q \gg 0$;

$\left(\mathrm{C} 2^{*}\right)$ for every $x \in R$, if $Q \in T_{I}(x)$ then there exists an integer $N$, possibly depending on $Q$, such that $Q^{N q} \subseteq\left(\left(I^{[q]}\right)^{*}: c x^{q}\right)_{Q}$ for all $q \gg 0$.

We shall soon use these conditions, in Theorem 3.5, to characterize precisely when tight closure commutes with localization for $I \subseteq R$. However, we need some preliminary results, as well as some new notation and terminology.

By a square locally stable test element $c$ we mean one such that $c=d^{2}$, where $d$ is a locally stable test element. For technical reasons that stem from Proposition 3.3(d), it is often advantageous to work with a square locally stable test element. We denote by $\sqrt{J}$ the radical of the ideal $J$ and by $\operatorname{Min}(J)$ the set of minimal primes of $J$.

Proposition 3.3. Let $R$ be a reduced Noetherian ring of positive prime characteristic $p$ with a locally stable test element. Let $I$ be an ideal of $R$, and let $x$ be an element of $R$.

(a) A prime ideal $Q$ of $R$ has the property that $x \notin\left(I R_{Q}\right)^{*}$ (in $\left.R_{Q}\right)$ if and only if $Q$ contains an ideal in $T_{I}(x)$. Hence, if localization commutes with tight closure for $I \subseteq R$, then $T_{I}(x)$ is the set of minimal primes of $I^{*}: R x$ and consequently, in this case, $T_{I}$ is the set of associated primes of $I^{*}$. In particular, if $I \subseteq R$ is such that localization commutes with tight closure, then all of the sets $T_{I}(x)$ and even $T_{I}$ itself are finite.

(b) $T_{I}(x)=T_{I^{*}}(x)$ and $T_{I}=T_{I^{*}}$.

(c) If $W$ is a multiplicative system in $R$, then the elements of $T_{I_{W}}(x / 1)$ (resp., $T_{I_{W}}$ ), working over $R_{W}$, are the expansions of those primes in $T_{I}(x)$ (resp., $T_{I}$ ) that do not meet $W$.

(d) If $c$ is a square locally stable test element then the sequence $\sqrt{I^{[q]}: c x^{q}}$ is nonincreasing as $q$ increases. Hence, if $Q \in \operatorname{Min}\left(I^{\left[q^{\prime}\right]}: c x^{q^{\prime}}\right)$ and $I^{[q]}$ : $c x^{q} \subseteq Q$ for some $q \leq q^{\prime}$, then $Q \in \operatorname{Min}\left(I^{[q]}: c x^{q}\right)$.

(e) If $c$ is a square locally stable test element and $P$ is a minimal prime of $I^{[q]}$ : $c x^{q}$, then $P$ contains an element of $T_{I}(x)$.

(f) If $c$ is a square locally stable test element, $T_{I}(x)$ is finite, and $q$ is so large that all elements of $T_{I}(x)$ are minimal over $I^{[q]}: c x^{q}$ (which is true for all $q \gg 0)$, then the minimal primes of $I^{[q]}: c x^{q}$ are precisely the elements of $T_{I}(x)$ and $\sqrt{I^{[q]}: c x^{q}}=\bigcap_{P \in T_{I}(x)} P$.

(g) $T_{I}(x)=\emptyset$ if and only if $x \in I^{*}$. Moreover, if $c$ is a test element then the condition that $I^{[q]}: c x^{q}=R$ for all $q \gg 0$ (resp., for all $q$ ) is also equivalent.

Proof. (a) Given any prime $P$ such that $x \notin\left(I R_{P}\right)^{*}$, every larger prime $Q$ has the same property, since there is a flat homomorphism $R_{Q} \rightarrow R_{P}$ and tight closure is persistent. Since $R$ has DCC on prime ideals, every such prime contains a minimal such prime. But the minimal primes with this property constitute $T_{I}(x)$, by part (5) of Proposition 3.1. The other statements in (a) are immediate. 
Parts (b) and (c) follow at once from the characterization of $T_{I}(x)$ in part (5) of Proposition 3.1.

For part (d), let $c=d^{2}$ where $d$ is a locally stable test element. It suffices to prove that $\sqrt{I^{[p q]}: c x^{p q}} \subseteq \sqrt{I^{[q]}: c x^{q}}$. Let $I^{[q]}: c x^{q} \subseteq P$ and assume that $I^{[p q]}: c x^{p q} \nsubseteq P$. Then $d^{2} x^{p q} \in I_{P}^{\left[q^{\prime}\right]}$. Hence $\left(d x^{q}\right)^{p} \in\left(I^{[q]}\right)_{P}^{[p]}$ and it follows that $d x^{q} \in\left(\left(I^{[q]}\right)_{P}\right)^{*}$. In particular, $d^{2} x^{q} \in\left(I^{[q]}\right)_{P}$, which means that $I^{[q]}: c x^{q} \nsubseteq$ $P-$ a contradiction. This proves the first assertion.

The second statement is immediate from the first. Suppose that $Q \in \operatorname{Min}\left(I^{\left[q^{\prime}\right]}\right.$ : $c x^{q^{\prime}}$ ) and $I^{[q]}: c x^{q} \subseteq Q$ for some $q \leq q^{\prime}$. If $Q$ is not minimal over $I^{[q]}: c x^{q}$, then there is a prime $P$ such that $I^{[q]}: c x^{q} \subseteq P \subseteq Q$ with $P \neq Q$. Then, from the first part, $P$ must also contain $I^{\left[q^{\prime}\right]}: c x^{q^{\prime}}$ and so $Q$ cannot be minimal over $I^{\left[q^{\prime}\right]}: c x^{q^{\prime}}$, either.

To prove (e), fix a minimal prime $P=P_{0}$ of $J_{q}=I^{[q]}: c x^{q}$. Since $P \supseteq \sqrt{J_{p q}}$, it contains a minimal prime $P_{1}$ of $J_{p q}$. Continuing in this way yields a sequence

$$
P_{0} \supseteq P_{1} \supseteq \cdots \supseteq P_{i} \supseteq \cdots
$$

such that $P_{i}$ is a minimal prime of $J_{q p^{i}}$ for every $i$. Since the prime ideals of a Noetherian ring have DCC, we can choose $n$ such that $P_{i}=P_{n}$ for all $i \geq n$, and it follows that $P_{n}$ is in $T_{I}(x)$ and is contained in $P$.

Part (f) is immediate because, once all of the finitely many primes in $T_{I}(x)$ are minimal primes of $I^{[q]}: c x^{q}$, part (e) shows that there cannot be any others.

To prove (g), note that we have by part (f) that if $T_{I}(x)=\emptyset$ and if one considers $J_{q}=I^{[q]}: c x^{q}$ using a square locally stable test element $c$, then the set of minimal primes of $J_{q}$ is empty for all $q$. This means that every $J_{q}=R$, which says that $c x^{q} \in I^{[q]}$ for all $q$, and this implies that $x \in I^{*}$. The other direction is clear. The last two conditions quite generally characterize when $x \in I^{*}$ for $c$ a test element.

Let $R$ be a reduced Noetherian ring of positive prime characteristic $p$, let $I$ be an ideal of $R$, and let $x, y \in R$. Let $P$ be a prime ideal in $T_{I}(x)$. We shall say that $y$ clears $P$ from $T_{I}(x)$ if $P$ is not in $T_{I}(x y)$. We shall say that the ideal $J \subseteq R$ clears $P$ from $T_{I}(x)$ if every element of $J$ clears $P$ from $T_{I}(x)$.

The following result gives some basic facts about clearing. Part $(\mathrm{g})$ is a bit different (though analogous) and will be needed in Section 4.

LEMMA 3.4 (Clearing Lemma). Let $R$ be a reduced Noetherian ring of positive prime characteristic $p$, let $I$ be an ideal of $R$, and let $x, y \in R$. Let c be a square locally stable test element for $R$.

(a) y clears $P \in T_{I}(x)$ if and only if $y^{q} / 1 \in\left(I^{[q]}: c x^{q}\right)_{P}$ for all $q \gg 0$. Hence, the set of all elements of $R$ that clear $P$ from $T_{I}(x)$ is an ideal contained in $P$.

(b) Condition (C2) or (C2*) for I and c implies that every prime $P$ of $T_{I}(x)$ has a power that clears $P$ from $T_{I}(x)$.

(c) Suppose that $P_{1}, \ldots, P_{n}$ are finitely many primes of $T_{I}(x)$ such that each $P_{i}$ has a power that clears $P_{i}$ from $T_{I}(x)$. Let $\mathcal{Q}$ be a family of primes $Q_{j}$ none 
of which contains any of the $P_{i}$. If the family $\mathcal{Q}$ is finite then there is an element $y$ of $\bigcap_{N} P_{i}^{N}$ for large $N$ not in any of the $Q_{j}$ that clears all of the $P_{i}$ from $T_{I}(x)$.

(d) With hypotheses as in (c) except that the family $\mathcal{Q}$ is countable, the same conclusion holds provided that $R$ has countable prime avoidance.

(e) Suppose that $T_{I}(x)$ and $T_{I}(x y)$ are finite. Then $\bigcap_{P \in T_{I}(x)} P \subseteq \bigcap_{P \in T_{I}(x y)} P$ and, if y clears at least one element of $T_{I}(x)$, the inclusion is strict.

(f) Every prime in $T_{I}(x y)$ contains a prime in $T_{I}(x)$.

(g) Let $(R, m)$ be local and let $z \in R$ be such that $z$ is not in an associated prime of $I^{[q]}$ except possibly $m$ and $z^{q} H_{m}^{0}\left(R / I^{[q]}\right)=0$ for all $q$. Then, for all $u \in R$ and all $q, m$ is not an associated prime of $I^{[q]}: u z^{q}$.

Proof. (a) The condition that $P$ not be in $T_{I}(x y)$ is simply that $P$ not be a minimal prime of $I^{[q]}: c(x y)^{q}$ for $q \gg 0$; since $P$ is minimal over $I^{[q]}: c x^{q}$ (which is smaller), this is equivalent to saying that $P$ does not contain $I^{[q]}: c(x y)^{q}$ for $q \gg 0$ (i.e., that $\left(I^{[q]}: c x^{q} y^{q}\right)_{P}=R_{P}$ for $q \gg 0$ ), which holds if and only if $y^{q} / 1 \in\left(I^{[q]}: c x^{q}\right)_{P}$ for $q \gg 0$. The second statement is then obvious.

Part (b) for condition (C2) is immediate from the definition. We may use (C2*) instead, because $T_{I^{*}}(x)=T_{I}(x)$ and $T_{I^{*}}(x y)=T_{I}(x y)$. Parts (c) and (d) are obvious.

The first statement in (e) follows from the fact that if $T_{I}(z)$ is finite then $\bigcap_{P \in T_{I}(z)} P=\sqrt{I^{[q]}: c z^{q}}$ for all sufficiently large $q$ by Proposition 3.3(f), taken with the obvious inclusion $I^{[q]}: c x^{q} \subseteq I^{[q]}: c x^{q} y^{q}$. The final statement is then obvious.

For part (f), note that any prime $P$ in $T_{I}(x y)$ contains the radical of $I^{[q]}: c(x y)^{q}$ for some $q$ and that this contains the radical of $I^{[q]}: c x^{q}$. Hence $P$ contains at least one minimal prime of $I^{[q]}: c x^{q}$, and each of these contains an element of $T_{I}(x)$ by Proposition 3.3(e).

For part (g) suppose that $m$ is associated to $I^{[q]}: u z^{q}$, and choose $y \notin I^{[q]}: u z^{q}$ such that $m y \subseteq I^{[q]}: u z^{q}$, that is, $m z^{q} y u \subseteq I^{[q]}$. Since $z^{q}$ is not in any associated prime of $I^{[q]}$ except $m$, it follows that if we take a primary decomposition of $I^{[q]}$ then $m y u$ is in the intersection $I^{\prime}$ of the primary components for primes other than $m$. Since $I^{\prime} / I^{[q]}$ is supported only at $m$, it follows that myu is killed by a power of $m$. This shows that $y u$ represents an element in $H_{m}^{0}\left(R / I^{[q]}\right)$ and so $z^{q} y u \subseteq I^{[q]}$, that is, $y \in I^{[q]}: u z^{q}$, a contradiction.

With these preliminary results, our characterization becomes straightforward.

THEOREM 3.5. Let $R$ be a reduced Noetherian ring of positive prime characteristic $p$ with a square locally stable test element $c \in R^{\circ}$, and let $I \subseteq R$ be an ideal. Then the following statements are equivalent.

(1) $\left(I_{W}\right)^{*}=\left(I^{*}\right)_{W}$ for all multiplicatively closed sets $W$ in $R$.

(2) Condition (C1) (or $\left(\mathrm{C}^{*}\right)$ ) holds for I and condition (C2) (or (C2*)) holds for I and $c$. 
Proof. We have already observed that (1) implies $\left(\mathrm{Cl}^{*}\right)$, which obviously implies (C1): see Proposition 3.3(a). Thus, we may ignore the parenthetical comment about $\left(\mathrm{Cl}^{*}\right)$ in the proof.

Assume (1) and suppose that $Q \in T_{I}(x)$. Then $Q$ is a minimal prime of $I^{*}: x$ and so we can chose $n \gg 0$ such that $Q^{n} \subseteq\left(I^{*}: x\right)_{Q}$, that is, $Q^{n}(x / 1) \subseteq\left(I^{*}\right)_{Q}$. Then, for all $q, c\left(Q^{n}\right)^{[q]} x^{q} \subseteq I_{Q}^{[q]}$, that is, $\left(Q^{n}\right)^{[q]} \subseteq\left(I^{[q]}: c x^{q}\right)_{Q}$. If $t$ exceeds the number of generators of $Q^{n}$, then $\left(Q^{n}\right)^{t q} \subseteq\left(Q^{n}\right)^{[q]}$ and so (C2) holds with $N=n t$. Hence, $(1) \Rightarrow(\mathrm{C} 1)$ and $(\mathrm{C} 2)$. Now $\left(\mathrm{C} 2^{*}\right)$ is obviously weaker than $(\mathrm{C} 2)$ and so to complete the proof it suffices to show that $(\mathrm{C} 1)$ and $\left(\mathrm{C} 2^{*}\right)$ together imply (1).

We assume otherwise and derive a contradiction. We may assume that $W=$ $R-Q$ for some prime ideal $Q$ by the results of Section 2 (or using the result of [AHH, Lemma 3.5, p. 79]). Suppose that $x \in\left(I_{Q}\right)^{*}$ but $x \notin\left(I^{*}\right)_{Q}$. We may assume that $x \in R$. By Noetherian induction, among all $x$ giving a counterexample there is one such that $\bigcap_{P \in T_{I}(x)} P$ is maximal. By Proposition 3.3(g), since $x \notin$ $I^{*}$ this is not the unit ideal; that is, $T_{I}(x)$ is not empty. Let $P \in T_{I}(x)$ be any element. Then $P$ is not contained in $Q$, by Proposition 3.3(a), since $x \in\left(I_{Q}\right)^{*}$; using $\left(\mathrm{C} 2^{*}\right)$, we know from Lemma 3.4(b) that $P^{N}$ clears $P$ from $T_{I}(x)$ for large $N$. Thus, we may choose $y \in P^{N}-Q$ such that $y$ clears $P$ from $T_{I}(x)$. Since $y$ is invertible in $R_{Q}$, we still have that $x y \notin I^{*} R_{Q}$ while $x y \in\left(I R_{Q}\right)^{*}$ is clear. But the intersection of the primes in $T_{I}(x y)$ is strictly larger than the intersection of those in $T_{I}(x)$, by Lemma 3.4(f), and this contradicts the hypothesis for the Noetherian induction.

REMARK 3.6. Let $R$ be as in Theorem 3.5, and suppose that condition (C2) holds in $R$ but that localization fails to commute with tight closure for $I \subseteq R$. The proof of Theorem 3.5 evidently shows that condition (C1) fails for $I$ and $x$ such that $x \in$ $\left(I_{Q}\right)^{*}-\left(I^{*}\right)_{Q}$ for some prime $Q$ of $R$.

\section{Growth of Frobenius Images and Localization}

In this section we focus our attention on the behavior of certain functions related to the Hilbert-Kunz function that we believe control the localization of tight closure in the local case. We shall informally say that "localization holds for $R$ " means that tight closure commutes with localization for all ideals of $R$.

As observed at the end of Section 2, to settle the localization problem for, say, excellent reduced local rings, it suffices to handle the complete case; we may even assume that the ring is complete with an uncountable residue field. Because localization holds if it holds modulo every minimal prime, it is sufficient to prove it for the case of a complete local domain.

A complete local domain $R$ is module-finite over a complete regular local ring $A$ with the same residue field. For sufficiently large $q$, the extension of fraction fields corresponding to the inclusion $A^{1 / q} \subseteq A^{1 / q}[R]$ will be separable. If we know that localization holds for $A^{1 / q}[R]$ then it holds for $R$, by Proposition 2.14 as extended in Remark 2.15. 
Therefore, it is reasonable to study the problem of localization of tight closure for a local domain $R$ module-finite over an excellent regular local ring $A$ and such that the extension of fraction fields is separable; we shall call such extensions generically étale. Since the case where the ring is complete implies all of the most important local cases, little is lost by assuming that countable prime avoidance holds. Likewise, as we shall see in Section 4.2, we may as well assume that there is a strong test ideal.

We shall attack the problem of localization in this situation in the main result, Theorem 4.5, of this section. Our goal is to show that, in a "minimal example" of the possible failure of tight closure to commute with localization and in the presence of a certain boundedness condition on behavior of local cohomology, localization becomes equivalent to an assertion about the asymptotic behavior of lengths of certain sequences of modules defined in terms of iterates of the Frobenius endomorphism. The length conditions, surprisingly, replace the finiteness conditions on the sets $T_{I}(x)$ discussed earlier.

We need several preliminaries.

DEFINITION 4.1. Let $R$ be a reduced Noetherian ring of positive prime characteristic $p$. If $(R, m)$ is local then we shall say that condition (LC) holds for $R$ if, for every ideal $J$, there is an integer $N$ such that $m^{N q} H_{m}^{0}\left(R / J^{[q]}\right)=0$ for all $q$ (evidently, if one knows this for all $q \gg 0$, it follows for all $q$ after enlarging $N$ if necessary).

If $I, x$ and a locally stable test element $c$ are fixed, then for each $Q \in T_{I}(x)$ we define

$$
\lambda_{q}(Q)=\frac{\lambda\left(R_{Q} /\left(I^{[q]}: c x^{q}\right)_{Q}\right)}{q^{\operatorname{dim}\left(R_{Q}\right)}},
$$

where $\lambda$ denotes length (in this case, over the local ring $R_{Q}$ ).

Definition AND Discussion 4.2. Let $R$ be a reduced Noetherian ring of positive prime characteristic $p$, and let $J$ be an ideal of $R ; J$ is called a strong test ideal for $R$ if $J$ meets $R^{\circ}$, and $J I^{*}=J I$ for every ideal $I$ of $R$. The main result of [Hu2] shows that there is such an ideal $J$, which is also a defining ideal for the singular locus in Spec $R$, provided that for every minimal prime $P$ of $R$, the singularities of $R / P$ can be resolved by blowing up an ideal that defines the singular locus. If blowing up such a $J_{0}$ resolves the singularities, then any high power of $J_{0}$ will be a strong test ideal. Such ideals are not unique, although there is a largest one.

By a very recent result [V1], if $R$ is a reduced local ring of positive prime characteristic $p$ such that $R$ has a completely stable test element, then if $(R, m)$ is complete or if the test ideal $\tau$ is $m$-primary (and in many other cases), $\tau$ is a strong test ideal!

Suppose that one has a strong test ideal $J$ with generators $j_{1}, \ldots, j_{k}$. Then, for every ideal $I$, if $u \in I^{*}$ then $u J \subseteq I J$ and we obtain the equations $u j_{s}=$ $\sum_{t=1}^{k} i_{s, t} j_{t}$. The usual determinantal trick for proving integral dependence on an ideal shows that $u$ is integrally dependent on $I$ using an equation of degree $k$, since 
$u$ is an eigenvalue of the $k \times k$ matrix $\left(i_{s, t}\right)$. This gives an extremely useful uniform bound on the degrees needed for equations displaying that elements in tight closures of ideals are in the integral closure of the ideal.

We refine the determinantal trick slightly. Suppose that $v$ and $u$ are elements of a domain $R$ and that $J \neq 0$ and $I^{\prime}$ are ideals such that $J u \in J\left(R v+I^{\prime}\right)=J v+J I^{\prime}$. Then we obtain that $u$ is an eigenvalue of a $k \times k$ matrix of the form $\left(r_{s, t} v+i_{s, t}^{\prime}\right)$, where the $r_{s, t} \in R$ and the $i_{s, t}^{\prime} \in I^{\prime}$. Let $V$ be a new variable. The characteristic polynomial of $\left(U I-\left(r_{s, t} V+i_{s, t}^{\prime}\right)\right)$ may be expanded as a polynomial of total degree $k$ in the variables $U$ and $V$ that is monic in $U$; the terms of degree smaller than $k$ have coefficients in $I^{\prime}$, which can be readily seen by thinking modulo $I^{\prime}$. Thus, the characteristic polynomial yields a degree- $k$ homogeneous polynomial $P(U, V)$ with coefficients in $R$, monic in $U$, such that $P(u, v) \in I^{\prime}$.

Before proving our main theorem, two preliminary lemmas are needed.

Lemma 4.3. Let $R$ be a reduced ring of prime characteristic $p$, module-finite and torsion-free over a regular domain $A$. Let $\mathcal{F}$ be the fraction field of $A$ and suppose that $\mathcal{F} \otimes_{A} R$ is étale (i.e. separable) over $\mathcal{F}$; that is, suppose $R$ is generically étale (or smooth) over A.

(a) For all $q, A^{1 / q}[R] \cong A^{1 / q} \otimes_{A} R$ is flat over $R$. Moreover, there exist elements $d \in A^{\circ}$ such that $d R \subseteq A\left[R^{p}\right]$ (equivalently, taking $p$ th roots, $d^{1 / p} R^{1 / p} \subseteq$ $\left.A^{1 / p}[R]\right)$.

(b) If $d \in A^{\circ}$ is chosen so that $d R \subseteq A\left[R^{p}\right]$, then

$$
d^{(q-1) / q} R^{1 / q} \subseteq A^{1 / q}[R] \text { for all } q \gg 0 .
$$

(c) If $c \in A^{\circ}$ is chosen so that $c R \subseteq A\left[R^{p}\right]$ (see (a)) then, for every ideal I of $R$ and all $q, q^{\prime}$, we have that

$$
\left(I^{\left[q q^{\prime}\right]}:_{R} c x^{q q^{\prime}}\right) \cap A \subseteq\left(I^{\left[q^{\prime}\right]}:_{R} c x^{q^{\prime}}\right)^{[q]} .
$$

(d) If $d \in A$ is such that $d R^{1 / q} \subseteq A^{1 / q}[R]$ then, for all $q^{\prime}, d^{q}\left(I^{\left[q q^{\prime}\right]}: x^{q q^{\prime}}\right) \subseteq$ $\left(I^{\left[q^{\prime}\right]}: x^{q^{\prime}}\right)^{[q]}$.

(e) Suppose, moreover, that $R$ has a strong test ideal $J$ with $k$ generators. Suppose that $d \in A^{\circ}$ is such that $d R \subseteq A\left[R^{p}\right]$. Let $c=d^{k}$, and let $c^{\prime} \in R^{\circ}$. Assume that there is an integer $N>0$ such that $c^{\prime} m^{N q} \subseteq\left(y^{q}\right)+\left(I^{[q]}:_{R} x^{q}\right)$ for all $q \gg 0$. Then there exists an integer $L>0$ such that, for all large $q$, $m^{N L q} \subseteq\left(y^{q}\right)+\left(I^{[q]}:_{R} c x^{q}\right)$.

Proof. Both statements in part (a) follow from Lemma 6.4 of [HH2, p. 50] and the discussion that immediately precedes it, where it is shown that if $c \in A^{\circ}$ is such that $R_{c}$ is étale over $A_{c}$ then $c$ has a power $c_{1}$ such that $c_{1} R^{1 / p} \subseteq A^{1 / p}[R]$. We may then take $d=c_{1}^{p}$.

(b) Write $q=p^{n}$. Let $h_{q}=\left(1+p+\cdots+p^{n-1}\right) / p^{n}$. We use induction on $n$ to prove that $d^{h_{q}} R^{1 / q} \subseteq A^{1 / q}[R]$. The case $n=1$ is immediate. Assume the result for $n$ and take $p$ th roots; this yields $d^{h_{n} / p} R^{1 / q p} \subseteq A^{1 / q p}\left[R^{1 / p}\right]$. Multiplying by $d^{1 / p}$ gives that 


$$
d^{\left(l+h_{n}\right) / p} R^{1 / q p} \subseteq A^{1 / q p}\left[A^{1 / p}[R]\right]
$$

(using the $n=1$ case). Since $\left(l+h_{q}\right) / p=h_{p q}$, we have completed the inductive step. Finally, $1+p+\cdots+p^{n-1}=(q-1) /(p-1) \leq q-1$, where $q=p^{n}$.

(c) Let $a \in I^{\left[q q^{\prime}\right]}: c x^{q q^{\prime}}$ be in $A$ as well, so that $a c x^{q q^{\prime}} \in I^{\left[q q^{\prime}\right]}$, and take $q$ th roots to obtain

$$
c^{1 / q} a^{1 / q} x^{q^{\prime}} \in I^{\left[q^{\prime}\right]} R^{1 / q} .
$$

It follows from part (b) that $c^{(q-1) / q} R^{1 / q} \subseteq A^{1 / q}[R]$ and so $a^{1 / q} c x^{q^{\prime}} \in I^{\left[q^{\prime}\right]} A^{1 / q}[R]$. From the flatness of $A^{1 / q}[R]$ over $R$ we then obtain that $a^{1 / q} \in I^{\left[q^{\prime}\right]}:_{R} c x^{q^{\prime}}$ (since $\left.a \in A, a^{1 / q} \in A^{1 / q} \subseteq A^{1 / q}[R]\right)$, and now we may take $q$ th powers to get the stated result.

(d) If $u x^{q q^{\prime}} \in I^{\left[q q^{\prime}\right]}$ then taking $q$ th roots yields that $u^{1 / q} x^{q^{\prime}} \in I^{\left[q^{\prime}\right]} R^{1 / q}$. Multiplying by $d$ then shows that $d u^{1 / q} x^{q^{\prime}} \in I^{\left[q^{\prime}\right]} A^{1 / q}[R]$ and so, with $B=$ $A^{1 / q}[R]$, we have $d u^{1 / q} \in I^{\left[q^{\prime}\right]} B:_{B} x^{q^{\prime}} B$; this is $\left(I^{\left[q^{\prime}\right]}:_{R} x^{q^{\prime}}\right) B$ because $B$ is flat over $R$, so $d u^{1 / q} \in\left(I^{\left[q^{\prime}\right]}:_{R} x^{q^{\prime}}\right) A^{1 / q} R$. Taking $q$ th powers yields the desired result.

(e) Consider an arbitrary element $z \in m$. For $q \gg 0$ and any $q^{\prime}$, we may replace $q$ by $q q^{\prime}$ and thus $c^{\prime} z^{N q q^{\prime}} \in\left(y^{q q^{\prime}}\right)+\left(I^{\left[q q^{\prime}\right]}:_{R} x^{q q^{\prime}}\right)$. Multiply by $d^{q}$ to obtain

$$
d^{q} c^{\prime} z^{N q q^{\prime}} \subseteq\left(d^{q} y^{q q^{\prime}}\right)+d^{q}\left(\left(I^{\left[q q^{\prime}\right]}: x^{q q^{\prime}}\right) \subseteq\left(d^{q} y^{q q^{\prime}}\right)+\left(I^{\left[q^{\prime}\right]}: x^{q^{\prime}}\right)^{[q]},\right.
$$

where we are using (b) to show that, by (d),

$$
d^{q}\left(I^{\left[q q^{\prime}\right]}: x^{q q^{\prime}}\right) \subseteq\left(I^{\left[q^{\prime}\right]}: x^{q^{\prime}}\right)^{[q]} .
$$

Since $c^{\prime}\left(d z^{N q^{\prime}}\right)^{q} \subseteq\left(d y^{q^{\prime}}\right)^{q}+\left(I^{\left[q^{\prime}\right]}: x^{q^{\prime}}\right)^{[q]}$ for all $q^{\prime}$ and all sufficiently large $q$, we have that $d z^{N q^{\prime}} \in\left(\left(d y^{q^{\prime}}\right)+\left(I^{\left[q^{\prime}\right]}: x^{q^{\prime}}\right)\right)^{*}$ for all $q^{\prime}$.

Because $J$ is a strong test ideal,

$$
J d z^{N q^{\prime}} \in J\left(d y^{q^{\prime}}\right)+J\left(I^{\left[q^{\prime}\right]}: x^{q^{\prime}}\right) .
$$

As in the final paragraph of Section 4.2, this yields that $P\left(d z^{N q^{\prime}}, d y^{q^{\prime}}\right) \in\left(I^{\left[q^{\prime}\right]}\right.$ : $\left.x^{q^{\prime}}\right)$, where $P(U, V)=U^{k}+r_{1} U^{k-1} V+\cdots+r_{n} V^{k}$ is a homogeneous polynomial of degree $k$ in two variables over $R$ monic in $U$. Factoring out $d^{k}=c$, we obtain that $c P\left(z^{N q^{\prime}}, y^{q^{\prime}}\right) \in\left(I^{\left[q^{\prime}\right]}: x^{q^{\prime}}\right)$ and hence that $P\left(z^{N q^{\prime}}, y^{q^{\prime}}\right) \in I^{\left[q^{\prime}\right]}: c x^{q^{\prime}}$ for all $q^{\prime}$. It follows that $z^{N k q^{\prime}} \in\left(y^{q^{\prime}}\right)+I^{\left[q^{\prime}\right]}: c x^{q^{\prime}}$ for all $q^{\prime}$. Set $L$ equal to $k$ times the minimal number of generators of $m^{N k}$. Then $m^{N L q^{\prime}} \subseteq\left(m^{N k}\right)^{\left[q^{\prime}\right]}$, and so $m^{N L q^{\prime}} \subseteq\left(y^{q^{\prime}}\right)+I^{\left[q^{\prime}\right]}: c x^{q^{\prime}}$ for all $q^{\prime}$.

Lemma 4.4 (Push-up Lemma). Let $(R, m)$ be an excellent local domain of positive prime characteristic $p$, let $I \subseteq R$, and let $x \in I$. Let $y$ be an element of $R$ that is not in any associated prime, with the possible exception of $m$, of either $(I+x R)^{[q]}$ or $I^{[q]}$ for any $q$. Let $P$ be a prime ideal in $T_{I}(x)$ of least height $h$, and let $J=I+y R$. If $h \leq \operatorname{dim} R-2$, then every minimal prime $Q$ of $P+y R$ is in $T_{J}(x)$.

Proof. Let $c$ be a square locally stable test element. It suffices to prove that $Q$ contains $J^{[q]}: c x^{q}$ for all large $q$ because, if so, then since $Q$ is minimal over 
$P+R y$ it will have to be minimal over $J^{[q]}: c x^{q}$ as well. To see this, note that if $Q^{\prime} \subset Q$ strictly is a prime containing $J^{[q]}: c x^{q}$ then $Q^{\prime}$ will contain some minimal prime over $I^{[q]}: c x^{q}$, and then $Q^{\prime}$ will contain some prime $Q^{\prime \prime}$ in $T_{I}(x)$. But the height of $Q^{\prime \prime}$ is at least $h$ and the height of $Q^{\prime}$ is at most $h$, so $Q^{\prime}=Q^{\prime \prime}$ is forced. But $y \in Q^{\prime}$ then gives a contradiction, as $y$ is not contained in any associated prime of $I^{[q]}$ except possibly $m$. Assume that $Q$ does not contain $\left((I, y)^{[q]}\right.$ : $\left.c x^{q}\right)$. Then $c x^{q} \in J_{Q}^{[q]}$ in $R_{Q}$, and we can write $c x^{q}-r_{q} y^{q} \in I_{Q}^{[q]}$ for some $r_{q} \in$ $R_{Q}$. Once we have localized at $Q$, which is properly contained in $m, y$ is not a zero divisor on $(I+x R)^{[q]}$, since it is outside the associated primes of $(I+x R)^{[q]}$ other than $m$. We therefore obtain that $r_{q} \in\left(I^{[q]}+x^{q}\right)_{Q}$. Thus, for some $s_{q} \in R_{Q}$ there is an equation

$$
x^{q}\left(c-s_{q} y^{q}\right) \in I_{Q}^{[q]} .
$$

This implies that $x \in\left(I_{Q}\right)^{*}$. (It suffices to work modulo a minimal prime of the completion to check this, and we may apply [HH3, Thm. 3.1]; the point is that the $q$ th root of the image of $c-s_{q} y^{q}$ will have order approaching 0.) But then $x \in$ $\left(I_{P}\right)^{*}$. Since $P \in T_{I}(x)$ this is a contradiction, which proves that $Q \in T_{J}(x)$.

We are now ready for the main result of this section.

THEOREM 4.5. Let $(R, m)$ be a local domain, with countable prime avoidance, that is module-finite and generically étale over an excellent regular local ring $A$. Suppose that $R$ has a strong test ideal $J \neq 0$ with $k$ generators. Suppose also that, for all $P \neq m, R_{P}$ has the property that the tight closure of ideals commutes with localization. Furthermore, assume that $R$ satisfies (LC) (see Definition 4.1). Fix a square locally stable test element $c$ that is the $k$ th power of an element of $A^{\circ}$ multiplying $R$ into $A\left[R^{p}\right]$, as in Lemma 4.3(e). Then the following are equivalent:

(1) tight closure of ideals in $R$ commutes with localization;

(2) for all ideals $I$ and all $x \in R$, there exists an $\varepsilon>0$ such that, for all $Q \in$ $T_{I}(x)$

$$
\ell(q, I, x, Q)=\lambda\left(\left(R_{Q} /\left(I^{[q]}: c x^{q}\right)_{Q}\right)\right)>\varepsilon q^{\operatorname{dim}\left(R_{Q}\right)}
$$

for all $q \gg 0$.

Proof. We first prove (1) $\Rightarrow$ (2). Assuming (1), Theorem 3.5 gives us that $T_{I}(x)$ is a finite set. Hence it suffices to prove that, for a fixed $Q \in T_{I}(x)$, $\lim \inf _{q} \lambda_{q}(Q)>0$.

Let $\mu$ denote the number of generators of $R$ as an $A$-module. Set $P=Q \cap A$, and let $W=A-P$. Let $s$ be the degree of the residue field extension $\left[R_{Q} / Q R_{Q}\right.$ : $\left.A_{P} / P A_{P}\right]$; note that, for $R_{Q}$-modules, length over $A_{P}$ is $s$ times the length over $R_{Q}$.

Since $x \notin\left(I_{Q}\right)^{*}$, we can choose $q^{\prime}$ such that $c x^{q^{\prime}} \notin I_{Q}^{\left[q^{\prime}\right]}$. Then:

$$
\begin{aligned}
& s \mu \cdot \ell(q, I, x, Q) \\
& \quad \geq \mu \cdot \lambda_{A_{P}}\left(A_{P} /\left(\left(I^{[q]}: c x^{q}\right)_{Q} \cap A_{P}\right) \geq \lambda_{A_{P}}\left(R_{W} /\left(\left(I^{[q]}: c x^{q}\right)_{Q} \cap A_{P}\right) R_{W}\right)\right. \\
& \quad \geq \lambda_{R_{Q}}\left(R_{Q} /\left(\left(I^{[q]}: c x^{q}\right)_{Q} \cap A_{P}\right) R_{Q}\right) \geq \lambda_{R_{Q}}\left(R_{Q} /\left(\left(I^{\left[q^{\prime}\right]}: c x^{q^{\prime}}\right)_{Q}\right)^{\left[q / q^{\prime}\right]}\right),
\end{aligned}
$$


by Lemma 4.3(c). But then $\ell(q, I, x, Q) \geq C q^{\operatorname{dim}\left(R_{Q}\right)}$ for some $C>0$ for large $q$, since $\lambda_{R_{Q}}\left(R_{Q} /\left(\left(I^{\left[q^{\prime}\right]}: c x^{q^{\prime}}\right)_{Q}\right)^{\left[q / q^{\prime}\right]}\right)$ is asymptotic to a positive constant times $\left(q / q^{\prime}\right)^{\operatorname{dim}\left(R_{Q}\right)}$ by the standard theory of Hilbert-Kunz functions; see [M]. This completes the " $\Rightarrow$ " direction.

We now consider the much more difficult converse direction. Assume the conditions in (2). Notice that, because we have assumed that localization holds for any proper localization of $R$, we know that condition (C2) holds for all $I, x, c$ (if $Q=m$ then we know the condition (LC), which is stronger). Suppose that the result is false, and choose $I$ maximal such that the tight closure of $I$ does not commute with localization. By Theorem 3.5 and Remark 3.6, there exists an element $x$ (and we may assume that $x \in\left(I_{Q}\right)^{*}-\left(I^{*}\right)_{Q}$ for some prime $Q$ ) such that either (C1) or (C2) fails. Since we know the latter, there must be such an $x$ with $T_{I}(x)$ infinite. Notice that, if we replace $x$ by $z x$ for any choice of $z \notin Q$, then the element $z x$ is still in $\left(I_{Q}\right)^{*}-\left(I^{*}\right)_{Q}$ and so $T_{I}(z x)$ is still infinite. We shall make several such replacements that will force increasingly controlled behavior on $T_{I}(z x)$. Each time, we change notation and write $x$ for what is really $z x$. We shall eventually obtain a contradiction.

First apply condition (LC) to choose $N$ such that $m^{N q}$ kills $H_{m}^{0}\left(R / I^{[q]}\right)$ for all $q$. Next, choose $z \in m^{N}$ not in $Q$ and not in any associated prime, except $m$, of any of the countably many ideals $I^{[q]}$. Then our previous remark shows that $T(z x)$ is still infinite. Moreover, $m$ is not associated to $I^{[q]}: u z^{q}$ for all $q$ and any choice of $u$ in $R$, by Lemma 3.4(g).

We replace $x$ by $z x$ and can assume from now on that $m$ is not associated to $I^{[q]}: c x^{q}$ for all $q$. Furthermore, this remains true if we again replace $x$ by $z^{\prime} x$ for some $z^{\prime}$.

We next replace $x$ by $z x$ for $z \notin Q$ so as to maximize the least height $h$ of a prime in $T_{I}(x z)$. Thus, without loss of generality we may assume that, for all $z \in$ $R-Q$, replacing $x$ by $z x$ does not clear all the primes of height $h$. Notice by Lemma 3.4(f) that, as we make such replacements, the least height occurring can only increase. Since (C2) holds it is immediate from the clearing lemma and our previous remark that there must be infinitely many primes of height $h$.

We shall next show that $h=d-1$. Assume $h \leq d-2$. We can choose an element $y$ of $m$ not in any associated prime (except possibly $m$ ) of any of the ideals $I^{[q]}$ and $(I+R x)^{[q]}$; then the push-up lemma shows that, for every prime $P$ of height $h$ in $T_{I}(x)$, every minimal prime $P^{\prime}$ of $P+y R$ is in $T_{(I+R y)}(x)$. But localization holds in $R_{P^{\prime}}$, so that only finitely many primes in $T_{I}(x)$ can lie inside $P^{\prime}$ (note that $T_{I}(x) \cap \operatorname{Spec}\left(R_{P^{\prime}}\right)=T_{I_{P^{\prime}}}(x)$; see Proposition 3.3(c)). On the other hand, the maximality of $I$ forces $T_{(I, y)}(x)$ to be finite also. Since we have shown that every one of the infinitely many primes in $T_{I}(x)$ of height $h$ lies inside some $P \in T_{(I, y)}(x)$, this contradiction proves that $h=d-1$.

Henceforth, we may assume that $h=d-1$. We choose $y$ sufficiently general as before. Recall that $c$ satisfies the hypothesis of Lemma 4.3(e); we shall need this below. We write $J^{\text {sat }}$ for the inverse image of $H_{m}^{0}(R / J)$ in $R$, that is, for the union of all the ideals $J: m^{N}$ as $N$ varies. 
By Noetherian induction, the ideal $(I, y)$ satisfies conditions $\left(\mathrm{C} 2^{*}\right)$ and $(\mathrm{C} 1)$. We know that the ideals $(I, y)^{[q]}: c x^{q}$ are either the whole ring or are $m$-primary (since $y$ is not in any of the primes of $T_{I}(x)$ ), and there exists an integer $N$ such that, for all $q, m^{N q} \subseteq(I, y)^{[q]}: c x^{q}$. Choose a general element $z \in m^{N q}$ and write $c z x^{q}-r_{q} y^{q} \in I^{[q]}$ for some $r_{q} \in R$. Then $r_{q} \in\left(I^{[q]}+\left(x^{q}\right)\right): y^{q} \subseteq\left(I^{[q]}+\left(x^{q}\right)\right)^{\text {sat }}$ by the choice of $y$. Hence the quotient $\left(\left(I^{[q]}+\left(x^{q}\right)\right): y^{q}\right) /\left(I^{[q]}+\left(x^{q}\right)\right)$ is contained in $H_{m}^{0}\left(R /\left(I^{[q]}+\left(x^{q}\right)\right)\right)$, and there exists an integer $B$ such that $m^{B q}$ annihilates this quotient. In particular, $m^{B q} r_{q} \in\left(I^{[q]}+\left(x^{q}\right)\right)$, and for general $u \in m^{B q}$ we can write

$$
\operatorname{czux} x^{q}-s_{q} x^{q} y^{q} \in I^{[q]}
$$

for some $s_{q} \in R$. Then $x^{q}\left(c z u-s_{q} y^{q}\right) \in I^{[q]}$ and it follows that

$$
c m^{(N+B) q} \subseteq\left(I^{[q]}: x^{q}\right)+\left(y^{q}\right)
$$

for all $q$.

We now apply Lemma 4.3(e) to conclude that there is a constant $D$ such that, for all $q$,

$$
m^{D q} \subseteq\left(y^{q}\right)+I^{[q]}: c x^{q} .
$$

In particular, $\lambda\left(R /\left(\left(y^{q}\right)+\left(I^{[q]}: c x^{q}\right)\right)\right) \leq \lambda\left(R / m^{D q}\right)$, and for large $q$ it follows that there is a constant $C>0$ such that

$$
\lambda\left(R /\left(\left(y^{q}\right)+\left(I^{[q]}: c x^{q}\right)\right) \leq C q^{d} .\right.
$$

Fix $\varepsilon>0$ as in the statement of the theorem. Let $N_{q}$ be the number of minimal primes above $I^{[q]}: c x^{q}$. We claim that

$$
N_{q} \leq C / \varepsilon
$$

where $C$ is as in the previous paragraph. Since every minimal prime above $I^{[q]}$ : $c x^{q}$ is also minimal above $I^{\left[q^{\prime}\right]}: c x^{q^{\prime}}$ for $q^{\prime} \geq q$ in our case, this means that the minimal primes above $I^{[q]}: c x^{q}$ stabilize for large $q$ and hence implies that $T_{I}(x)$ is a finite set.

Recall that $m$ is not associated to $I^{[q]}: c x^{q}$. The associativity formula for multiplicities gives that

$$
\lambda\left(R /\left(\left(y^{q}\right)+I^{[q]}: c x^{q}\right)=e\left(y^{q} ; R /\left(I^{[q]}: c x^{q}\right)\right)=q e\left(y ; R /\left(I^{[q]}: c x^{q}\right)\right)\right.
$$

and

$$
q e\left(y ; R /\left(I^{[q]}: c x^{q}\right)\right)=q\left(\sum_{P \in \operatorname{Min}\left(I^{[q]}: c x^{q}\right)} e(y ; R / P) \lambda\left(R_{P} /\left(I^{[q]}: c x^{q}\right)_{P}\right)\right)
$$

and hence

$$
C q^{d-1} \geq \sum_{P \in \operatorname{Min}\left(I^{[q]}: c x^{q}\right)} \lambda\left(R_{P} /\left(I^{[q]}: c x^{q}\right)_{P}\right)=\sum_{P \in \operatorname{Min}\left(I^{[q]}: c x^{q}\right)} l(q, I, x, P) .
$$

Thus $C q^{d-1} \geq N_{q} \cdot \varepsilon q^{d-1}$ and hence $N_{q} \leq C / \varepsilon$, as claimed. 


\section{Further Remarks and Questions}

Discussion 5.1 (Finding Specific Test Exponents). Tight closure is known to commute with localization in many specific cases: under mild conditions on the ring, this is true for ideals generated by monomials in parameters and ideals $I$ such that $R / I$ has finite phantom projective dimension. We refer the reader to [AHH, Sec. 8] for a detailed discussion of various results. The known results on when tight closure commutes with localization therefore imply the existence of test exponents for many ideals. However, little is known about how to determine a specific test exponent for a given ideal $I$. We want to raise this as a problem. If one has a specific test exponent for $c, I$, then to test whether $u \in I^{*}$ one need only test whether $c u^{q} \in I^{[q]}$ for that one value of $q$. We believe that the best hope for giving a useful algorithm for testing when an element is in the tight closure of an ideal lies in this direction. It would be of considerable interest to solve the problem of determining test exponents effectively even for parameter ideals.

Discussion 5.2 (Algorithmic Testing for Tight Closure). We want to point out that in certain instances there is an algorithm, in a technical sense, for testing whether specific elements are in a tight closure. We do not believe that this particular method will ever be implemented. In any given instance where it may be applied, it does eventually terminate, showing that the specific element $x$ either is or is not in the tight closure of $I$. However, we do not have a way of estimating a priori how long testing may need to go on before the algorithm terminates.

The method may be applied to ideals $I$ such that the tight closure of $I$ is the same as the plus closure of $I$. We review the latter notion. Suppose that $R$ is a domain. If $I \subseteq J \subseteq R$ and there is an integral extension (equivalently, a module-finite extension) $S$ of $R$ with $J \subseteq I S$, then $J \subseteq I^{*}$. If $R^{+}$denotes the integral closure of $R$ in an algebraic closure of its fraction field (the absolute integral closure; see [HH4] and [S] for further discussion), we can let $I^{+}=I R^{+} \cap R$ and then $I \subseteq I^{+} \subseteq I^{*}$. Is $I^{*}=I^{+}$? Except in trivial cases where $I=I^{*}$ for all $I$, we do not know whether this is always true in any normal domain. By a hard theorem (cf. [S]) it is true for parameter ideals, and a result of Aberbach [A] permits one to extend this to ideals $I$ such that $R / I$ has finite phantom projective dimension. The point we want to make is that for ideals such that $I^{*}=I^{+}$, which may well be all ideals, there is an algorithm of sorts.

Let $R$ be a countable Noetherian domain of prime characteristic $p>0$ in which basic operations can be performed algorithmically, with a known test element $c$, and such that one can test algorithmically for membership in an ideal in polynomial rings over $R$, for example, a finitely generated domain over a finitely generated field.

FACT. If $R$ is as just described and $I \subseteq R$ satisfies $I^{*}=I^{+}$, then one can test algorithmically whether $y \in R$ is in $I^{*}$. In particular, one has such a test if $R$ is an affine domain over a finitely generated field and if I is generated by monomials in elements $z_{1}, \ldots, z_{d}$ generating an ideal of height $d$. 
Here is the idea of the algorithm: one can effectively enumerate all the algebras $S_{1}, \ldots, S_{n}, \ldots$ that are module-finite over $R$. Alternately, test whether $y \in I S_{n}$ and whether $c y^{p^{n}} \in I^{\left[p^{n}\right]}$. If $y \in I^{*}=I^{+}$then the former test eventually succeeds; if $y \notin I^{*}$, the latter test eventually fails.

Of course, this method is awful: this algorithm only gives emphasis to the problem of effective determination of test exponents.

Discussion 5.3 (Uniform Test Exponents). Let $R$ be reduced and finitely generated over an excellent local ring. So far as we know, it is possible that for a given locally stable test element $c$ there exists a test exponent valid for all ideals $I$ simultaneously. It would suffice to give such an exponent for all $m$-primary tightly closed ideals as $m$ varies, and even for those that are maximal with respect to the property of being tightly closed and not containing a given element of the ring, since every tightly closed ideal is an intersection of such ideals.

A more modest question that seems more approachable is whether, given $c$, there exists a single test exponent for all ideals containing a given $m$-primary ideal $J$, because then one can construct a moduli space for the set of ideals.

Discussion 5.4. It is reasonable to ask whether localization can be proved for suitable local domains (e.g., those that are excellent, have countable prime avoidance, and are generically étale over a regular local ring) if, for all ideals $I \subseteq R$ and all $x \in R$, there exist constants $\varepsilon$ and $\delta$ greater than zero such that, for all $q$ and for all $Q \in T_{I}(x), \delta q^{\operatorname{dim}\left(R_{Q}\right)} \geq \ell(q, I, x, Q) \geq \varepsilon q^{\operatorname{dim}\left(R_{Q}\right)}$. If localization holds then the first inequality can be deduced from (C2); the second was shown in the proof of Theorem 4.5 to follow from $(\mathrm{C} 1)$. Thus, these conditions are necessary. It also seems reasonable to ask whether $\ell(q, I, x, Q) / q^{\operatorname{dim}\left(R_{Q}\right)}$ approaches a (necessarily positive) limit as $q \rightarrow \infty$.

\section{References}

[A] I. M. Aberbach, Tight closure in F-rational rings, Nagoya Math. J. 135 (1994), 43-54.

[AHH] I. M. Aberbach, M. Hochster, and C. Huneke, Localization of tight closure and modules of finite phantom projective dimension, J. Reine Angew. Math. 434 (1993), 67-114.

[B] L. Burch, Codimension and analytic spread, Proc. Cambridge Philos. Soc. 72 (1972), 369-373.

[He] J. Herzog, Ringe der Characteristik $p$ und Frobeniusfunktoren, Math Z. 140 (1974), 67-78.

[H1] M. Hochster, Tight closure in equal characteristic, big Cohen-Macaulay algebras, and solid closure, Contemp. Math., 159, pp. 173-196, Amer. Math. Soc., Providence, RI, 1994.

[H2] — Solid closure, Contemp. Math., 159, pp. 103-172, Amer. Math. Soc., Providence, RI, 1994.

[HH1] M. Hochster and C. Huneke, Tight closure and strong F-regularity, Mem. Soc. Math. France (N.S.) 38 (1989), 119-133. 
[HH2] - Tight closure, invariant theory, and the Briançon-Skoda theorem, J. Amer. Math. Soc. 3 (1990), 31-116.

[HH3] - Tight closure and elements of small order in integral extensions, J. Pure Appl. Algebra 71 (1991), 233-247.

[HH4] - Infinite integral extensions and big Cohen-Macaulay algebras, Ann. of Math. (2) 135 (1992), 53-89.

[HH5] - F-regularity, test elements, and smooth base change, Trans. Amer. Math. Soc. 346 (1994), 1-62.

[HH6] - Tight closure in equal characteristic zero, preprint.

[Hu1] C. Huneke, Tight closure and its applications, CBMS Regional Conf. Ser. in Math., 88, Amer. Math. Soc., Providence, RI, 1996.

[Hu2] - Tight closure and strong test ideals, J. Pure Appl. Algebra 122 (1997), 243-250.

[Hu3] - Saturation of Frobenius powers of ideals, Comm. Algebra (to appear).

[K1] M. Katzman, Some finiteness properties of the Frobenius endomorphism and their applications to tight closure, Ph.D. dissertation, Univ. of Michigan, Ann Arbor, 1994.

[K2] - Finiteness of $\bigcup_{e}$ Ass $F^{e}(M)$ and its connections to tight closure, Illinois J. Math. 40 (1996), 330-337.

[Ku] E. Kunz, Characterizations of regular local rings of characteristic p, Amer. J. Math. 91 (1969), 772-784.

[LS] G. Lyubeznick and K. E. Smith, Strong and weak F-regularity are equivalent for graded rings, Amer. J. Math. 121 (1999), 1279-1290.

[McD] M. McDermott, Tight closure, plus closure and Frobenius closure in cubical cones, Ph.D. dissertation, Univ. of Michigan, Ann Arbor, 1996.

[M] P. Monsky, The Hilbert-Kunz function, Math. Ann. 263 (1983), 43-49.

[S] K. E. Smith, Tight closure of parameter ideals, Invent. Math. 115 (1994), 4160.

[V1] A. Vraciu, Tight closure and local cohomology, Ph.D. dissertation, Univ. of Michigan, Ann Arbor, 2000.

[V2] - Local cohomology of Frobenius images over graded affine algebras, J. Algebra (to appear).

M. Hochster

Department of Mathematics

University of Michigan

Ann Arbor, MI 48109-1109

hochster@math.lsa.umich.edu
C. Huneke

Department of Mathematics

University of Kansas

Lawrence, KS 66045

huneke@math.ukans.edu 